apuntesuniversitarios.upeu.edu.pe

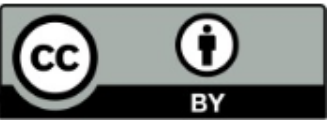

Apuntes Universitarios, 2020: 10(3), julio-setiembre

ISSN: 2304-0335 DOI: https://doi.org/10.17162/au.v10i3.464

\title{
Epistemología y educación: ciencias de la educación e investigación educativa desde una mirada epistemológica
}

\author{
Epistemology and education: learning sciences and educational research from an \\ epistemological perspective \\ María Gisella Boarini, ${ }^{1 a}$ Analía Inés Portela, ${ }^{2}$ y María Elisa Di Marco ${ }^{3}$ \\ Universidad Nacional de Cuyo (UNCuyo), Mendoza, Argentina ${ }^{123}$ \\ Universidad de Congreso, Mendoza, Argentina ${ }^{2}$ \\ (iD) Orcid ID: https://orcid.org/0000-0001-6388-2766 ${ }^{1}$ \\ Orcid ID: https://orcid.org/0000-0003-2517-4448 \\ (iD) Orcid ID: https://orcid.org/0000-0003-4972-9348
}

Recibido: 26 de diciembre de 2019

Aceptado: 1 de marzo de 2020

\section{Resumen}

En el presente trabajo se realiza un análisis de los fundamentos epistemológicos sobre los que se ha cimentado el campo educativo desde su origen hasta nuestros días, a fin de comprender en profundidad la situación actual de la investigación educativa. Para ello, se lleva a cabo un estudio histórico-epistemológico que procura develar la justificación que, en cada momento y contexto, ha fundamentado dicho campo. Este recorrido muestra que, en primer lugar, la historicidad (referida a lo contextual, no-racional, no-científico) de la ciencia de la educación fue dejada de lado deliberadamente para garantizar el desarrollo de una ciencia racional. En segundo lugar, y debido a la persistencia de ciertos problemas epistemológicos, esta historicidad es entendida como un valor y se convierte en el fundamento disciplinar. El trabajo argumenta que el uso de la historicidad como criterio de justificación (ya sea para eliminarla como para utilizarla) no es criterio suficiente para fundamentar el campo. Se requiere un criterio que trascienda los límites de la disciplina misma y permita hacer frente a los problemas relativos a la historicidad y a la consecuente inconmensurabilidad.

Palabras clave: Ciencias de la educación; investigación sobre la educación; filosofía de la educación; historicidad; inconmensurabilidad.

\footnotetext{
${ }^{\mathrm{a} C}$ Correspondencia a la autora: giseboarini@yahoo.com.ar
} 


\begin{abstract}
In the present paper an analysis of the epistemological foundations on which the educational field has been founded since its origin until today is made, in order to understand in depth the current situation of educational research. To this end, a historical-epistemological study is carried out. This study seeks to uncover the justification that, in each moment and context, has founded the said field. This path shows that, at first, historicity (referred to the contextual, non-rational, nonscientific) was deliberately set aside to guarantee the development of a rational science. Later, and due to the persistence of certain epistemological problems, this historicity is understood as a value and becomes the disciplinary foundation. The work argues that the use of historicity as a criterion of justification (either to eliminate it or to use it) is not sufficient criterion to base the field. It requires a criterion that transcends the limits of the discipline itself and allows to face the problems related to historicity and consequent incommensurability.
\end{abstract}

Keywords: Learning sciences; educational research; educational philosophy; historicity; incommensurability

\title{
Introducción
}

El campo de las ciencias de la educación aparece como un prometedor espacio de reflexión y enriquecimiento en torno a lo específicamente educativo; no obstante, presenta ciertas inconsistencias, como la falta de acuerdos en torno al objeto propio del campo y a los límites disciplinares, el poco impacto práctico de la investigación educativa o el cuestionamiento acerca de su especificidad teórica y práctica. Por ello, se considera conveniente realizar un análisis que permita comprender los criterios epistemológicos que fundamentan a las ciencias de la educación, no en la resolución de los problemas expresados, sino a fin de entender mejor la situación actual del campo.

La metodología utilizada en este trabajo es histórico-epistemológica. Se desarrolla el origen y evolución histórica de la disciplina, desde la pedagogía científica del siglo XIX hasta la investigación educativa actual. Cabe señalar que no se intenta hacer una historia integral de la disciplina, sino que se busca señalar los criterios epistemológicos que, en cada momento histórico, fundamentaron el campo. Por ello, esta exposición no es meramente descriptiva: en cada período se realiza una reflexión crítica y valorativa que permite vislumbrar futuras investigaciones. A fin de favorecer esta comprensión se recurre a la bibliografía utilizada en cada momento histórico; por tal motivo, en los primeros apartados se encontrarán mayormente publicaciones de hace algunas décadas y en los últimos se apreciará la bibliografía más reciente. 


\section{La pedagogía científica a principios del siglo XIX}

La educación, como práctica, es tan antigua como el hombre mismo. Sin embargo, hacia fines del siglo XVIII surgieron algunos planteamientos que procuraron darle fundamentación científica. Si bien la palabra pedagogía es utilizada con anterioridad, existe acuerdo en la comunidad académica en considerar a Juan Federico Herbart como el iniciador de la pedagogía científica (Bowen, 2001; Huarte Cuéllar, 2012; Kanz, 2001, entre otros).

En 1806, Herbart publica su libro Pedagogía general derivada del fin de la educación, con el que se propone brindar a pedagogos y preceptores un ensayo que permita reducir la espontaneidad y la intuición en la tarea educativa. En efecto, allí sienta las bases de la pedagogía como ciencia, fundamentándola en la ética o filosofía práctica y la psicología. La primera otorga a la pedagogía el conocimiento de los fines del hombre y, por lo tanto, del fin de la educación (la moralidad). La segunda brinda el conocimiento de los medios para conseguir dichos fines ${ }^{\mathrm{i}}$. Si bien este filósofo postula la necesidad de una reflexión pedagógica independiente, reconoce la dependencia de esta reflexión con respecto a la filosofía (Huarte Cuéllar, 2012, p. 26).

La novedad introducida por Herbart, que marca un nuevo rumbo en la teoría pedagógica, radica en su intencionalidad de justificar el conocimiento educacional. En efecto, para que la educación no sea algo improvisado debe fundamentarse en teorías pedagógicas seguras, basadas en conclusiones y principios científicos confiables, derivados de la ética y la psicología.

La propuesta herbartiana de una pedagogía científica es continuada en la tradición alemana de las ciencias del espíritu. Aunque la pedagogía va tomando diferentes tintes según los autores y contextos, hay una continuidad en la intención de fundamentarla científicamente. El fundamento que se esgrima para ello irá cambiando a través del tiempo, pero permanecerá la necesidad de la justificación científica del conocimiento pedagógico.

\section{La historicidad dejada de lado: ciencia de la educación y ciencias de la educación}

Cabe mencionar brevemente el concepto de criterio de demarcación científica. Éste alude a los elementos o rasgos epistémicos que los investigadores definen en cada momento para distinguir el conocimiento científico del que no lo es, a fin de "identificar las características epistémicas o metodológicas que separan las creencias científicas de las no-científicas" (Laudan, 
1983, p. 118). En el caso de la pedagogía, el criterio de demarcación utilizado hasta comienzos del siglo XX consiste en los principios filosóficos que dan sustento al conocimiento pedagógico. A partir de este momento se produce un cambio radical en dicho criterio: se da inicio a la tradición positivista dentro del campo educativo y se empieza a hablar de ciencia de la educación. El sociólogo francés Emilio Durkheim es quien introduce este cambio al oponerse al concepto de pedagogía por su conexión con la filosofía. Su intención fue transformar la ciencia de la educación en una ciencia positiva, basada en hechos, que permita describir la realidad educativa como hecho social y estudiarla al modo de las ciencias naturales, esto es, describiendo sus causas físicas y observables. En palabras del propio Durkheim: "Dado que el principio causal se ha verificado en los diferentes campos de la naturaleza, de tal modo que ha ido extendiendo progresivamente su dominio de lo físico-químico a lo biológico, y de aquí al terreno psicológico, es justo suponer que de igual modo será válido también en el campo de lo social" (citado en Schoeck, 1977, p. 239).

El criterio de demarcación que separa a esta ciencia de la educación de otras aproximaciones no-científicas es la verificabilidad de los enunciados. La base sobre la que debe construirse la ciencia es la observación; a partir de allí, se inducen las leyes que rigen los fenómenos educativos y que permiten predecir nuevos fenómenos. Así se distingue lo científico de lo metafísico (en cuanto histórico, valorativo, no-racional, no-científico) y se pretende eliminar esto último del terreno de la ciencia. Dicho de otro modo, la historicidad -o lo históricoes dejada deliberadamente de lado en defensa de una ciencia racional.

Esta tradición positivista va impregnando todas las áreas del saber y, poco a poco, se comienzan a aplicar los resultados provenientes de otras disciplinas a la educación, así es como empieza a utilizarse el plural de ciencias de la educación. Quizás, las ciencias que primeramente se aplican son la sociología y la psicologíaii Sin embargo, en pocos años comienzan a hacerlo muchas otras que se van consolidando como líneas de trabajo con programas de investigación propios. De este modo, hacia mediados del siglo XX el "estudio científico de la educación" se amplió hasta albergar todos aquellos saberes a los que empezó a llamarse "Ciencias de la educación” (García Amilburu y García Gutiérrez, 2012, p. 27); estas comprenden: política educativa, orientación educativa, filosofía de la educación, educación comparada, historia de la educación, teoría de la educación, biología de la educación, organización escolar, economía de la educación. 
A raíz de la explosión y difusión del nuevo campo, en numerosas universidades, libros, revistas y congresos se sustituye el nombre de pedagogía por el de "ciencias de la educación." Este cambio de denominación expresa "una tendencia hacia la positividad científica y, consecuentemente, hacia la desvinculación de la filosofía, ámbito con el que el saber pedagógico ha mantenido, desde su origen, una clara y profunda dependencia” (Escolano, 1978, p. 19).

\section{Intentos de unificación o integración del campo}

Esta proliferación de ciencias, y su consecuente disparidad y falta de unidad, comienza a generar preocupaciones que se concretizan en diversos intentos por unificar las ciencias de la educación como campo teórico. Surgen así dos propuestas: por lado, la de una ciencia integradora (pedagogía o teoría de la educación, según el caso) y, por otro, diversas clasificaciones que procuran distinguirlas y establecer relaciones mutuas. Sin embargo, ninguno de estos intentos llega a cumplir su cometido. El planteo de la pedagogía como aquella capaz de integrar los resultados de las diferentes ciencias educativas fracasa porque, justamente, la tradición de ciencias de la educación es radicalmente opuesta a la de pedagogía por su conexión con la filosofía (metafísica).

La sugerencia de la teoría de la educación como ciencia integradora (Quintana Cabanas, 1995) se frustra, pues lo importante no es debatir acerca de la clase de teoría sobre la que debería basarse la teoría de la educación, sino preguntarse si la teoría educacional debe definirse por referencia a un conjunto de disciplinas o, contrariamente, por la práctica educacional, que es su objeto (Wilfred Carr, 1990, p. 43).

Por otro lado, las propuestas de clasificación son numerosas (por ejemplo, Fernández y Sarramona, 1964; García Hoz, 1968) y todas ellas suponen que la unidad de las ciencias de la educación estaría garantizada por "la convergencia de todas las ciencias que entran en juego en el análisis de la educación, que vendría a constituirse en el objeto aglutinante" (Escolano, 1978, p. 22). No obstante, terminan fracasando ya que pretenden hacer complementarias ciencias epistemológicamente diferentes u opuestas. Por tal motivo, a partir de 1970 el modelo disciplinar comienza a ser el centro de serias polémicas, principalmente de parte de pedagogos influidos por la Escuela de Frankfurt. 


\section{Críticas al modelo disciplinar}

En este contexto, Carr (1990) señala ciertos conflictos entre las distintas ciencias de la educación producidos porque, en realidad, no se busca dar solución a problemas educativos. Por ejemplo, al problema práctico de hacer que los niños trabajen en clase se lo transforma en un problema para la filosofía de la educación: mostrar la suficiencia o no de determinada teoría psicológica - por ejemplo, el conductismo- para explicar el aprendizaje. Al respecto ironiza:

Así, mientras que la clase de "análisis filosófico de la teoría conductista y sus suposiciones" (...) puede tener éxito en mostrar a otros filósofos la irrelevancia de la psicología, la psicología de la educación sigue con una firme convicción de la irrelevancia de la filosofía. Y cuando la sociología de la educación pueda entrar en acción, toda esta filosofía y psicología serán despachadas como un absurdo ideológico que simplemente sirve a los intereses de algún grupo social dominante. Ahora bien, si la psicología no acepta lo que la filosofía dice acerca de su marco teórico, y la sociología rechaza tanto la filosofía como la psicología como meras "legitimaciones" para ideas dominantes, ¿cómo se supone que opera esta clase de estudio teórico de problemas educacionales? Claramente a los que se enfrentan a la resolución de estos problemas se les están ofreciendo no sólo consejos diferentes sino también conflictivos. (pp. 60-61)

Como consecuencia de estas reflexiones pueden identificarse dos problemas epistemológicos presentes en el modelo de las ciencias de la educación: historicidad e inconmensurabilidad

\section{Historicidad e inconmensurabilidad en las ciencias de la educación}

A partir de los años 60 se produce el conocido "giro historicista"; autores como Thomas Kuhn (2004 [1962]) y Paul Feyerabend (1989 [1962]; 1986 [1975]) empiezan a considerar que los elementos históricos -en sus aspectos circunstanciales, valorativos, de cosmovisiones, etc.han determinado efectivamente la ciencia (en un sentido fuerte o moderno). Es decir, los elementos metafísicos que la epistemología clásica ${ }^{\text {iii }}$ se empeñó en eliminar de la ciencia a fin de conferirle certeza y validez, son los que han guiado y fundado esa misma ciencia. Tanto Kuhn como Feyerabend terminan denunciando la inconmensurabilidad en la que cae la ciencia al intentar eliminar los elementos metafísicos de ella:

(...) el problema de la Inconmensurabilidad es la tesis según la que dos teorías científicas resultan incomparables o, al menos, imperfectamente comunicables o traducibles entre sí dado que carecen de metro o patrón común, extrínseco a ellas. Por lo 
mismo, no existirían criterios racionales para afirmar que una es mejor o más verdadera que la otra. Aunque existen antecedentes, en 1962 coincidieron Thomas Kuhn y Paul Feyerabend en la postulación de esta tesis. (...) y llegan a similares conclusiones: las grandes teorías científicas son inconmensurables entre sí, y ni el recurso a experimentos ni la argumentación lógica son criterios válidos que permitan una justa comparación entre ellas. (Gelonch Villarino, 2010, p. 579)

Ahora bien, si se vuelve la mirada sobre el campo de las ciencias de la educación, se observa que ha sido su historicidad la que orientó su conformación como campo científico. El término historicidad aquí introducido alude a lo históricamente condicionado, en contraposición con lo que tiene pretensión de validez universal por estar legitimado por una verdad supratemporal (Grondin, 2002, pp. 32-33).

En efecto, como ya se dejó en claro, fueron aspectos coyunturales (aparición de libros, revistas, cátedras) y valorativos (creencias epistemológicas, concepciones metodológicas) los que definieron a las nuevas disciplinas. Si bien las ciencias de la educación procuraron eliminar los elementos metafísicos de su fundamentación científica, estos elementos son los que terminaron justificándolas. En otras palabras, no fue la existencia de un objeto común y compartido el criterio que orientó su conformación, sino su misma historicidad; ni fue la observación la que justificó determinada teoría o ciencia de la educación, sino que fue la creencia en que la educación es un hecho observable y medible y que, por lo tanto, sólo puede ser estudiado de modo empírico ${ }^{\mathrm{iv}}$.

En consecuencia, dado que la historicidad -que pretendió ser dejada de lado para conferir cientificidad a las ciencias de la educación- fue la que orientó y fundamentó la configuración de las mismas, se explica con claridad la inconmensurabilidad que existe entre las ciencias educativas. No hay una ciencia que determine qué es la educación y qué aspectos de ella le corresponde estudiar a cada disciplina. Por ende, el educador no puede integrar en su práctica los conocimientos aportados por las distintas ciencias de la educación, ya que muchas de ellas son inconmensurables entre sí. Por este motivo, se torna difícil establecer un diálogo entre ellas. Este diálogo no es imposible, pero a fin de realizarla, se debe comenzar por alejarse de los términos metodológicos y científicos y luego enfocarse en la relevancia de los aspectos "metafísicos" u “ontológicos” que fundamentan a las ciencias de la educación. 


\section{La historicidad como valor epistémico: ciencia crítica de la educación y epistemologías contemporáneas}

Queda en claro que, hacia el último tercio del siglo pasado, entra en crisis el relato de "la racionalidad científica, vertida en ideología de cientificismo” (Hoyos Medina, 2010, p. 13). En efecto, "el ideal de la modernidad empezó a dar señales de agotamiento hasta finalmente ser desplazado por la visión escéptica de la posmodernidad. Conforme transcurría el siglo pasado, eran cada vez más evidentes las exiguas capacidades de la razón para construir el ideal de una sociedad de paz y armonía" (Montero, 2014, p. 83). El trasfondo de esta transformación es una desilusión cada vez más evidente por las promesas incumplidas del ideal de progreso indefinido:

Dos grandes guerras mundiales fueron golpes devastadores contra la modernidad. El hombre había alcanzado, a través de la razón, las cumbres de la ciencia y la tecnología, pero también la humanidad se descubría a sí misma en los umbrales del infierno de un holocausto nuclear. La razón había llevado a la humanidad a conquistas extraordinarias en la ciencia, pero esas conquistas, lejos de llevarlo al ideal de la sociedad perfecta, se volvían contra el propio hombre. Con todo su adelanto científico, el hombre no había avanzado un milímetro en la construcción de una sociedad que garantice la felicidad y la plenitud al ser humano. Un sentimiento de desilusión estremece el corazón de la humanidad. Este sentimiento es una respuesta casi exclusivamente emocional a los ideales frustrados de la modernidad. Esa respuesta moldea lo que se ha dado en llamar la sociedad posmoderna. (Montero, 2014, p. 83)

En este sentido, como los esfuerzos del modelo disciplinar por salvar su cientificidad no pudieron cumplir su objetivo, hacia fines del siglo $\mathrm{XX}$, la historicidad se convierte en un valor epistémico y, sobre todo, en el criterio de fundamentación de la ciencia crítica de la educación y de las epistemologías contemporáneas.

\section{Ciencia crítica de la educación}

A partir de la década de 1970 irrumpe la filosofía crítica en el ámbito educativo (Vázquez, 1994, p. 19). Surgen así diferentes tendencias, sintetizadas por Méndez Pardo y Méndez Pardo (2007) en tres grandes líneas: la pedagogía crítica de Apple, McLaren y Giroux (Peters y Giroux, 2012), la teoría crítica de la educación de Carr y Kemmis (Carr, 1990; Hirst y Carr, 2005) y la reflexión sudamericana que critica al neoliberalismo educativo (Achili, 2008; Sirvent, 2007). Todas ellas toman como fuente los planteos de la Escuela de Frankfurt -principalmente, de Habermas- y buscan consolidar una ciencia crítica de la educación. 
Para dicha ciencia la teoría es crítica de la realidad. Vázquez (1994) explica que la finalidad de la crítica es provocar una ilustración de los grupos (una autoconciencia respecto de las presiones internas y externas para lograr un sujeto autónomo) y, seguidamente, la transformación de las condiciones sociales existentes (p. 20). De esto se sigue que todo conocimiento está condicionado por las circunstancias histórico-sociales.

El objeto de la ciencia crítica de la educación es la práctica educativa, entendida no como un simple hacer, sino como algo construido subjetiva, social, histórica y políticamente (Kemmis en Carr, 1990, p. 15). Por esto mismo, los significados atribuidos o auto-comprensiones de los sujetos involucrados son una parte esencial de la teoría educacional.

La fundamentación epistemológica de la ciencia crítica de la educación puede caracterizarse a partir de las siguientes hipótesis: a) la teoría no es el resultado de la investigación científica sino que guía todo su proceso; b) el núcleo de la ciencia no es "la justificación racional aplicable a sus productos finales, sino los rasgos racionales de los procesos del descubrimiento científico" (Carr, 1990, p. 119); c) la racionalidad científica no consiste en acomodarse a unas reglas metodológicas rígidas, sino en lograr una aceptabilidad racional acerca de qué teorías hay que aceptar y cuáles rechazar; d) la teoría, en cuanto práctica social, se justifica en la esfera pública mediante el debate y mediante los acuerdos y desacuerdos de un grupo en determinadas circunstancias socio-históricas; dicho de otro modo, la teoría se legitima a partir del consenso racional; e) los valores impregnan toda la tarea científica, la ciencia no es desinteresada ni neutra: "la ideología tratará de explicar determinados sucesos sobresalientes que atraviesa una comunidad (...) será la encargada de seleccionar y organizar los propósitos y establecer las prioridades" (Expósito, 2018, p. 33).

Esta perspectiva ha realizado importantes aportes a la investigación educativa. En primer lugar, recupera el papel de los elementos valorativos, históricos y contextuales en la tarea científica. Esto significa una superación con respecto a la teorización anterior, que pretendía ser imparcial y completamente objetiva pero que, en realidad, sólo conseguía escindir unos determinados valores epistémicos y éticos sin permitir, por lo mismo, su discusión o adhesión libre y consciente. Esta recuperación trae consecuencias positivas en la investigación educativa; por un lado, la abre al plano ético y político, volviendo a poner en escena una discusión tan propia como fundamental para la educación. En segundo término, revaloriza la voz de los practicantes, de los maestros y alumnos. Esta puesta en valor de la experiencia de quienes están 
continuamente en la práctica educacional ha contribuido a la estimación de su tarea y de su rol educativo. También ha ayudado a sacar del silencio y el ocultamiento la coerción ejercida por diversas formas de poder que promovían la permanencia de prácticas no conducentes al mejoramiento de las personas involucradas en la acción educativa.

Sin embargo, la ciencia crítica de la educación presenta algunas limitaciones. Primeramente, cabe destacar su justificación a partir del consenso racional. Para este enfoque, la utilización del consenso como criterio de cientificidad lleva a la emancipación de la influencia de lo dado y lo impuesto, conduce a la autonomía racional y genera una auto-consciencia y una acción ilustrada. Como se observa, la justificación de la ciencia sigue siendo inherente al científico; ya no es la comprobación empírica o la deducción lógica la que otorga calidad de científica a una teoría, sino los acuerdos grupales. Sin embargo, continúa siendo el sujeto y su modo de aproximarse al objeto los que garantizan la validez científica. La realidad no es el criterio de verdad, sino que lo es la actitud del científico, la que ha ido cambiando a lo largo del tiempo, pero sigue enfocada en el sujeto cognoscente. Esto conlleva a una circularidad argumentativa en la que el mismo sujeto que hace ciencia determina qué es científico y qué no lo es, a partir del modo en que ese mismo sujeto se aproxima al objeto. Esta circularidad surge por no recurrir a elementos que trasciendan las definiciones que se producen dentro de la misma disciplina.

Por otro lado, la historicidad, que en la tradición empirista pretendió ser dejada de lado por considerarse que atentaba contra la calidad de científica de una teoría, en la epistemología crítica es tomada como un valor. En efecto, son las interpretaciones subjetivas, las cosmovisiones, los valores éticos y políticos, y las circunstancias socio-históricas particulares las que confieren validez educacional a la teoría educativa. Es más, la teoría no es más que la sistematización de esas interpretaciones subjetivas y juicios de valor. Por lo tanto, la teoría o ciencia educacional no pueden decir nada más acerca de la educación que esas mismas autocomprensiones. Lo que confiere cientificidad a la teoría educacional es la autonomía racional con que se develan y se explicitan esas auto-comprensiones. Asimismo, su carácter esencialmente situado impide generalizaciones y teorías de validez universal, pero ella misma se presenta como tal. Una ciencia que pretende eliminar todo a priori termina siendo ella misma un presupuesto incuestionable. 
Siegel (2012) reduce al absurdo la cuestión mediante el conocido argumento aristotélico: si se sostiene que no hay verdad objetiva y se considera que esta afirmación es una verdad objetiva, se está contradiciendo; por otro lado, si se acepta que tal afirmación es relativa al tiempo y lugar, no habría argumentos racionales para aceptarla como verdadera; lo denomina relativismo autorrefutante (p. 30). Por ello, al justificarse la ciencia educacional a partir de su historicidad, sólo puede teorizar acerca de las auto-comprensiones de los practicantes y está limitada para "decir algo" sobre el hombre que valga para todos; entonces, es incapaz de juzgar qué es lo mejor para el educando y el educador en una situación. Al final, el único criterio al que se puede apelar es el consenso grupal.

\section{Epistemologías contemporáneas}

Pueden distinguirse en las investigaciones actuales dos grandes tipos de fundamentación epistemológica: la empirista y la constructivista o contextualista. Ya ha sido explicada la epistemología empirista en educación, por lo tanto, sólo se tratará aquí la constructivista. Dentro de ésta hay un gran abanico de propuestas entre las que se distinguen la investigación-acción (Denzin y Lincoln, 2000; Méndez Pardo y Méndez Pardo, 2007; Vasilachis de Gialdino, 2009); diversidad epistemológica - o epistemología multicultural o multiculturalismo- (Hesse-Biber, 2014; Siegel, 2012); investigación cualitativa, triangulación y copresencia de paradigmas de investigación educativa (Newman et al., 2012; Vasilachis de Gialdino, 2011; Yeasmin y Ferdousour Rahman, 2012); contextualismo o constructivismo epistemológico (Martín, 2007).

Estos enfoques contemporáneos poseen puntos de encuentro. En primer lugar, la teoría es siempre contextual, no se dispone de una teoría educacional objetiva e independiente de los sujetos participantes porque no existen fundamentos del conocimiento exteriores al sujeto. En consecuencia, el conocimiento se justifica a partir del consenso racional.

En segundo lugar, se plantea la existencia de una diversidad epistemológica o variedad de modos de conocer. El lenguaje, los mitos, las expresiones artísticas, la historia de cada grupo se proponen como los criterios de verdad. Se postula que no existen criterios externos a los sujetos mismos que permitan comparar y juzgar sus conocimientos, por lo que deben respetarse todos ellos como igualmente legítimos. El problema que salta a la vista es el de la inconmensurabilidad.

Lo mismo sucede al hablar de copresencia de paradigmas de investigación. Como cada paradigma define desde sí y para sí la realidad, la ciencia, y la investigación, no se cuenta con 
criterios que trasciendan esos mismos paradigmas que permitan el diálogo y la complementariedad.

Con respecto a la diversidad epistemológica, es absolutamente necesario tratar a los miembros de otros grupos con respeto: una mente respetuosa "es la que responde a las diferencias entre grupos e individuos con la misma bondad y compromiso. Implica tolerancia, respuesta activa y asertiva ante los problemas" (Krumm, 2015, p. 125). Sin embargo, el trato respetuoso implica tomar en serio esas ideas y someterlas a discusión racional, no implica necesariamente considerarlas tan verdaderas como cualquier otra. En otras palabras, pluralismo, no relativismo (Siegel, 2012, p. 74). Vale para el campo educativo lo que Dellacasa (2016) postula para la antropología: "afirmaba una multiplicidad ficticia, escasamente comprometida, puesto que en definitiva arriesgaba bastante poco y no ponía en juego nada `esencial” (p. 41). Además, la diversidad propuesta y el reclamo por una legitimidad de las epistemologías alternativas es, en realidad, un reclamo por la inclusión de minorías o grupos excluidos. Más que tratarse de epistemología, se trata de una cuestión de justicia (Siegel en Code et al, 2012, p. 122).

En tercer lugar, la epistemología contextualista afirma que el objeto disciplinar se construye a partir del campo disciplinar y, por otro lado, que el campo se construye en referencia al objeto. En otras palabras, "el objeto disciplinar no puede establecerse en forma a priori, sino que, en tanto objeto científico, sólo puede constituirse como un enfoque teórico construido desde las mismas investigaciones desarrolladas en el campo" (Martín, 2007, 13). Esta argumentación circular deja en evidencia que la historicidad como fundamento de la conformación disciplinar es limitada. Los postulados propuestos por una ciencia que se determina a sí misma sólo pueden ser válidos para el pequeño contexto socio-cultural e histórico en el que surgen. Y, por lo tanto, de igual modo queda limitada su cientificidad. Esta circularidad no puede superarse a menos que se acuda a un criterio que trascienda los límites de la disciplina y a partir del cual pueda juzgarse qué pertenece al campo y cómo estudiarlo.

En cuarto lugar, se propone una noción unívoca de ciencia, ya que la misma disciplina determina su objeto y su criterio de demarcación: "si la Ciencia de la Educación tiene un objeto propio, éste lo constituyen los rasgos del enfoque que asumen los investigadores vinculados con la disciplina, un enfoque teórico-epistemológico que regula y condiciona, desde el discurso peculiar de la disciplina, las producciones dentro del campo" (Martín, 2007, p. 46). 
Es decir que, por un lado, se reconoce la coexistencia de enfoques y paradigmas y se proclama la legitimidad de todos ellos. Pero por otro, únicamente se aceptan como propios de la disciplina aquellos que se corresponden con el "enfoque que asumen los investigadores". En última instancia, como los investigadores son quienes determinan el enfoque teóricoepistemológico que regula el campo disciplinar, se sigue que son ellos quienes determinan qué es legítimo y qué no lo es. Este problema podría comenzar a salvarse si se acudiera a criterios de cientificidad que fueran más allá de la práctica misma de investigación.

\section{Conclusiones}

Este trabajo expuso los criterios epistemológicos que justificaron el campo educativo en los distintos momentos de su evolución y dejan en evidencia que estos criterios han sido insuficientes para lograrlo. Primeramente, durante la vigencia de los enfoques de ciencia y ciencias de la educación, la disciplina—o, más tarde, el modelo disciplinar — se concentró en eliminar la historicidad de la esfera de la ciencia para garantizar un progreso de la misma. Esta concepción de ciencia presentó dos grandes inconvenientes: en primer lugar, la historicidad asumida como lo metafísico o no-racional- que quiso expulsarse, terminó justificando a las ciencias de la educación. Esta situación trajo aparejado el problema de la inconmensurabilidad entre dichas ciencias.

En un segundo momento, se mostró cómo esa historicidad, que anteriormente se había evitado, ahora era asumida como el fundamento de la ciencia de la educación, dando lugar a la ciencia crítica de la educación y a las epistemologías contemporáneas constructivistas o contextualistas. Sin embargo, dado que esta ciencia ha desconocido referencia alguna a criterios que superen las definiciones dentro de la disciplina, ha caído en los mismos enunciados apriorísticos y universales que criticaba.

En efecto, los criterios de cientificidad han cambiado, ya no es la verificación empírica sino el consenso racional lo que legitima la teoría, pero continúa siendo el mismo investigador el que determina de modo a priori qué es científico y qué no lo es, de acuerdo con sus asunciones con respecto al objeto y a la disciplina. En otras palabras, el carácter situado y contextual de la ciencia crítica impide generalizaciones y teorías de validez universal. Sin embargo, ella misma se presenta como una teoría de validez universal que no cuestiona sus fundamentos. 
En conclusión, todas las propuestas de ciencia o ciencias de la educación desde el siglo XIX hasta la actualidad han procurado justificar dicha ciencia; es decir, buscar un criterio que garantice su validez y certeza. El criterio utilizado ha ido cambiando, pero siempre ha estado centrado en la historicidad: para evitarla o para asumirla. Este criterio, como se ha dejado en claro, es insuficiente para fundar una ciencia educativa. Por ello, una posibilidad para sortear este abismo y poder traspasar los límites propios de cada teoría o disciplina es volver la discusión al plano metafísico-antropológico. Esto no implica obtener acuerdos inmediatos ni verdades infalibles, pero seguramente los debates serán más fructíferos y se estará llevando la discusión en una dirección más promisoria.

\section{Referencias bibliográficas}

Achilli, E. (2008). Formación docente e interculturalidad. Diálogos pedagógicos. 6(12), 121-138. Recuperado de http://revistas.bibdigital.uccor.edu.ar/index.php/prueba/article/view/471

Bowen, J. (2001). Historia de la Educación Occidental. Tomo III: El occidente moderno. Europa y el Nuevo Mundo. Siglos XVII-XX. Barcelona, España: Herder.

Carr, W. (1990). Hacia una Ciencia Critica de la Educacion. Introducción de Stephen Kemmis. Barcelona, España: Laertes.

Code, L. et al. (2012). Epistemological Diversity: A Rountable. En C. Ruitenberg \& D. C. Phillips (Eds.), Education, Culture and Epistemological Diversity: Mapping a Disputed Terrain (pp. 121-143). New York, USA: Springer.

Coll, C.; Palacios, J. y Marchesi, A. (1990). Desarrollo psicológico y educación. 2. Psicología de la Educación. Madrid, España: Alianza.

Dellacasa, M. A. (2016). Repensar la antropología ante la crisis de la modernidad: el culto a la unidad de lo diverso. Revista San Gregorio, 15(2), 34-43. Recuperado de http://revista.sangregorio.edu.ec/index.php/REVISTASANGREGORIO/article/view/255

Denzin, N. \& Lincoln, Y. (Eds.) (2000). Handbook of Qualitative Research. London: Sage Publications.

Erazo Santander, O. A. (2012). Reflexiones sobre la psicología educativa. Revista de Psicología GEPU, 3(2), 139-157. Recuperado de https://revistadepsicologiagepu.es.tl/REFLEXIONES-SOBRE-LA-PSICOLOG\%CDA- 
Escolano, A. (1978). Las ciencias de la educación. Reflexiones sobre algunos problemas epistemológicos. En A. Escolano et al. Epistemología y educación (pp. 9-11 y 15-26). Salamanca, España: Sígueme.

Expósito, C. (2018). De la reflexión ideológica a la realidad pedagógica. Apuntes Universitarios. Revista de Investigación, 8(2), 31-47. DOI: http://dx.doi.org/10.17162/au.v8i2.192

Fernández, A. y Sarramona, J. (1964). La educación: Constantes y Problemática Actual. Perú: CEAC.

Feyerabend, P. K. (1986). Tratado contra el método: Esquema de una teoría anarquista del conocimiento. Madrid, España: Tecnos.

Feyerabend, P. K. (1989). Límites de la ciencia: Explicación, reducción y empirismo. Barcelona, España: Paidós.

García Amilburu, M. y García Gutiérrez, J. (2012). Filosofía de la educación. Cuestiones de hoy y de siempre. Madrid, España: Narcea-UNED.

García Hoz, V. (1968). Principios de Pedagogía Sistemática. Madrid, España: Rialp.

Gelonch Villarino, S. (2009). Ciencia, Metafísica y Filosofía. O para una nueva Unidad del Saber, en Ciencia y Tecnología. En J.M. Torres y J.E. Calderón (Comps.), Reflexiones Humanistas. Mendoza, Argentina: EDIUNC.

Gelonch Villarino, S. (2010). Voz "Inconmensurabilidad”. En A. González García (Ed.), Diccionario de filosofía. Pamplona, España: EUNSA.

Grondin, J. (2002). Introducción a la hermenéutica filosófica. Prólogo de H. G. Gadamer (2a ed.). Barcelona, España: Herder.

Guerrero Serón, A. (1996). Manual de Sociología de la Educación. Madrid, España: Síntesis.

Herbart, J. F. (1935). Pedagogía general derivada del fin de la educación. Prólogo de Ortega y Gasset (Trad. L. Luzuriaga). Madrid, España: Espasa Calpe.

Hesse-Biber, S. (Ed.) (2014). Feminist Research Practice. London, UK: SAGE.

Hirst, P. \& Carr, W. (2005). Philosophy and Education: A Symposium. Journal of Philosophy of Education, 39(4), 615-632.

Hoyos Medina, C. (2010). Epistemología y objeto pedagógico: ¿es la pedagogía una ciencia? (3 ${ }^{\mathrm{a}}$ ed.). Madrid, España: Plaza y Valdés. 
Huarte Cuéllar, R. (2012). Kant y Herbart: dos visiones de la Pedagogía como ciencia entre los siglos XVIII y XIX. Fermentario, 6, 1-34. Recuperado de: http://www.fermentario.fhuce.edu.uy/index.php/fermentario/article/viewFile/95/52

Kanz, H. (2001). Immanuel Kant. UNESCO: Oficina Internacional de Educación. Recuperado de: http://www.ibe.unesco.org/publications/ThinkersPdf/kants.pdf. [El texto original se publicó en 1993 en Perspectivas: revista trimestral de educación comparada, 23(3/4), 837-854].

Kenklies, K. (2012). Educational Theory as Topological Rhetoric: The Concepts of Pedagogy of Johann Friedrich Herbart and Friedrich Schleiermacher. Studies in Philosophy \& Education, 31(3), 265-273. DOI: 10.1007/s11217-012-9287-6

Krumm, S. (2015). Motivar, transmitir, transformar: una revisión de las competencias necesarias para el oficio de enseñar. Apuntes Universitarios. Revista de Investigación, 5(1), 119-134. DOI: http://dx.doi.org/10.17162/au.v0i1

Kuhn, T. (2004). La estructura de las revoluciones científicas. ( $8^{\mathrm{a}}$ reimpresión). Buenos Aires, Argentina: Fondo de Cultura Económica.

Laudan, L. (1983). The Demise of the Demarcation Problem. En R. S. Cohen \& L. Laudan (Eds.), Physics, Philosophy and Psychoanalysis: Essays in Honor of Adolf Grunbaum (pp.111-127). Dordrecht, Holland: Reidel.

Martín, A. V. (2007). El status epistemológico y el objeto de la Ciencia de la Educación (Tesis doctoral). Facultad de Filosofía, Humanidades y Artes, San Juan, Argentina.

Méndez Pardo, A. y Méndez Pardo, S. (Coords.) (2007). El docente investigador en educación: Textos de Wilfred Carr. Chiapas, México: Universidad de Ciencias y Artes de Chiapas.

Montero, R. (2014). El desafío de la posmodernidad para el cristiano. Apuntes Universitarios. Revista de Investigación, 4(1), 79-96. DOI: http://dx.doi.org/10.17162/au.v0i1

Newman, I.; Ridenour, C.; Newman, C.; Smith, S. \& Brown, R. (2012). Detecting Low Incidents Effects: The Value of Mixed Methods Research Designs in Low-N Studies. Mid-Western Educational Researcher, 25(4), 31-46.

Putnam, H. (1975). Mathematics, Matter and Method. Philosophical Papers, vol. 1. London, UK: Cambridge University Press.

Peters, M. \& Giroux, H. (2012). Henry Giroux on Democracy Unsettled: From Critical Pedagogy to the War on Youth. Interview by Michael A. Peters. Geopolitics, History, and 
International Relations, 4(1), 156-174. Recuperado de: https://www.ceeol.com/search/article-detail?id=175733

Quintana Cabanas, J. M. (1995). Teoría de la educación. Concepción antinómica de la educación. Madrid, España: Dykinson.

Rodríguez Sedano, A. (2006). Hacia una fundamentación epistemológica de la pedagogía social. Educación y educadores, 9(2), 131-147. Recuperado de http://educacionyeducadores.unisabana.edu.co/index.php/eye/article/view/667

Schoeck, H. (1977). Historia de la sociología. Barcelona, España: Herder.

Siegel, H. (2012). Epistemological Diversity and Education Research: Much Ado About Nothing Much? En C. Ruitenberg \& D. C. Phillips (Eds.), Education, Culture and Epistemological Diversity: Mapping a Disputed Terrain (pp. 65-83). New York, USA: Springer.

Sirvent, M. T. (2007). Conferencia dada en la Pre-Jornada de Acción Participativa en Investigación, Bahía Blanca, Argentina. Recuperado de: https://www.youtube.com/watch?v=4M-_TVc9mCw

Thorndike, E. L. (1903) Educational Psychology. New York, USA: The Science Press.

Vasilachis de Gialdino, I. (2009). Los fundamentos ontológicos y epistemológicos de la investigación cualitativa. Forum: Qualitative Social Research, 10(2), 1-26. Recuperado de: http://www.qualitative-research.net/index.php/fqs/article/view/1299/2778

Vasilachis de Gialdino, I. (2011). De las nuevas formas de conocer y de producir conocimiento. En N. Denzin \& Y. Lincoln, Manual de Investigación Cualitativa. Vol. I. El campo de la Investigación Cualitativa (pp. 11-22). Barcelona: Gedisa. Recuperado de: http://www.ceil-conicet.gov.ar/2011/09/nuevo-capitulo-de-las-nuevas-formar-de-conocery-de-producir-conocimiento-por-irene-vasilachis/

Vázquez, S. M. (1994). La teoría del curriculum en la actualidad. Buenos Aires, Argentina: CIAFIC.

Vázquez, S. M. (2001). La Filosofía de la Educación: Estado de la cuestión y líneas esenciales. Buenos Aires, Argentina: CIAFIC.

Yeasmin, S. \& Ferdousour Rahman, K. (2012). “Triangulation” Research Method as the Tool of Social Science Research. BUP Journal, 1(1), 154-163. Recuperado de: https://www.bup.edu.bd/journal/154-163.pdf 
' Para ampliar acerca de la concepción herbartiana de pedagogía, ver Kenklies (2012).

ii Piénsese en el movimiento de la Educational Sociology, en Estados Unidos, a partir de la década de 1920 y en la publicación de Thorndike Educational Psychology, de 1903.

Para ampliar información sobre el origen y desarrollo de la sociología de la educación, ver Guerrero Serón (1996);

Rodríguez Sedano (2006). Sobre la psicología de la educación, ver; Coll, Palacios y Marchesi (1990); Erazo Santander (2012).

iii Por epistemología clásica se entiende lo que Putman (1975) denominara concepción heredada.

iv Cf. Gelonch Villarino (2009). 